\title{
Multiplicity and oscillations in a model for catalyzed oxidation of carbon monoxide
}

\author{
M. C. Lemos and A. Córdoba \\ Departamento de Física de la Materia Condensada, Universidad de Sevilla, Apartado 1065, 41080 Sevilla, Spain
}

(Received 16 November 1993)

\begin{abstract}
We extend a model proposed for explaining multiplicity and oscillations of concentrations and temperature in catalyzed oxidation of carbon monoxide; the importance of the dimension of the system and the closure approximation applied to the results, and, especially to the oscillatory behavior, is analyzed. Kinetic phase transitions, namely, single state $\leftrightarrow$ multiplicity, single state $\leftrightarrow$ oscillations, and multiplicity $\leftrightarrow$ oscillations are found, depending on the reaction heat and the temperature relaxation parameter. Also, the role played by desorption of reactants is considered. When there is no desorption, temperature oscillations take place around room temperature, but if desorption is operative, oscillations occur about a higher temperature. For the one-dimensional case a spurious kinetic phase transition is obtained when the singlet closure approximation is applied.
\end{abstract}

\section{INTRODUCTION}

Multiplicity and oscillations are phenomena often observed in reactions catalyzed on a surface. Oxidation of carbon monoxide on platinum or other metals is one of the most representative of these reactions and has been the object of a great number of experimental and theoretical studies. A number of models, based on different mechanisms, have been proposed for explaining multiplicity and oscillations in this reaction, easy models as well as models with increasing difficulty. ${ }^{1}$

Recently some papers have analyzed kinetic phase transitions, bistability, hysteresis, "poisoning," and so on, for this or similar reactions, on the basis of lattice models, using the Monte Carlo method or applying different closure approximations to obtain rate equations. ${ }^{2-15}$

On the other hand, the importance of the dimension of the system and the approximation applied for obtaining the kinetic equations from a master equation has been revealed in the study of kinetic phase transitions. Specifically, closure approximations that are too simple can give rise to spurious kinetic phase transitions in onedimensional systems where competitive adsorption of two species takes place, with or without further chemical reaction, when there is interaction between nearest neighbors $^{16,17}$ (something similar to that happens in the equilibrium Ising model when the Bragg-Williams or the Bethe-Peierls approximations are applied). Significant differences among results obtained using different approximations have been detected in oscillatory situations. ${ }^{18}$

Moreover, models where, because of reaction heats, a catalytic surface can keep a different temperature from that of its surroundings and temperature oscillations can originate jointly with concentration oscillations have been considered. ${ }^{19-21}$ Temperature fluctuations give rise to a strong nonlinearity of kinetic equations owing to the Arrhenius law.

In this paper, the model initially proposed by Lagos, Sales, and Suhl ${ }^{19}$ is extended to consider other features of the problem and to analyze the influence of the system dimension and the kind of closure approximation on the re- sults and especially on oscillatory behavior and its characteristics.

In Sec. II the two-dimensional model is analyzed by applying a singlet closure approximation (SCA), and in Sec. III desorption of reactives is also considered; in Sec. IV a doublet closure approximation (DCA) is applied; finally, in Sec. $\mathrm{V}$ the case of a linear chain is studied for comparison with the two-dimensional system, the influence of the system dimension in this kind of kinetic phase transition being made clear.

\section{SQUARE LATTICE. SINGLET CLOSURE APPROXIMATION}

Temperature and concentration oscillations in chemical reactions catalyzed on a surface constitute a representative case of oscillatory states in far from thermodynamic equilibrium systems. Catalyzed oxidation of carbon monoxide is a good example of this kind of behavior.

A known model, by Lagos, Sales, and Suhl, ${ }^{19}$ assumes that the reaction takes place according to three elemental mechanisms:

$$
\begin{aligned}
& \mathrm{O}_{2}(g)+2 \mathrm{~V}(s) \rightarrow 2 \mathrm{O}(s), \\
& \mathrm{CO}(g)+V(s) \rightarrow \mathrm{CO}(s), \\
& \mathrm{CO}(s)+\mathrm{O}(s) \rightarrow \mathrm{CO}_{2}(g)+2 V(s),
\end{aligned}
$$

where $\mathrm{O}_{2}(g), \mathrm{CO}(g)$, and $\mathrm{CO}_{2}(g)$ indicate, respectively, oxygen, carbon monoxide, and carbon dioxide molecules in gaseous phase; $\mathrm{O}(s)$ and $\mathrm{CO}(s)$ denote an oxygen atom and a carbon monoxide molecule adsorbed on the surface and $V(s)$ is a vacant site on the surface. This simple scheme constitutes the Langmuir-Hinshelwood (LH) mechanism.

The surface where adsorption and reaction processes take place is assumed to be like a square lattice and each oxygen atom $(\mathrm{O})$ and each carbon monoxide molecule (CO) can occupy one lattice site at most. Adsorption of an oxygen molecule $\left(\mathrm{O}_{2}\right)$ needs two adjacent vacant sites, dissociation in two atoms being allowed. On the other hand, a $\mathrm{CO}$ molecule and an $\mathrm{O}$ atom, both adsorbed next 
neighbors, can react, constituting a $\mathrm{CO}_{2}$ molecule, which leaves the surface immediately. The transition probabilities for the processes $(2.1)-(2.3)$ are chosen as

$$
W_{1}=k_{1}(T) n_{v v}, \quad W_{2}=k_{2}(T) n_{v}, \quad W_{3}=k_{3}(T) n_{\mathrm{O}-\mathrm{CO}},
$$

where $k_{i}(T)(i=1,2,3)$ denote the rate constants of the processes $(2.1)-(2.3) ; n_{V}$ is the vacant fraction, $n_{V V}$ the vacant pair fraction, and $n_{\mathrm{O}-\mathrm{CO}}$ the density of oxygen-carbon monoxide pairs.

Kinetic equations for the singlet densities $n_{\mathrm{O}}$ and $n_{\mathrm{CO}}$ are formulated from a mean field approximation, where all the cluster densities are written in terms of singlet densities (SCA)

$$
\begin{aligned}
\frac{d n_{\mathrm{O}}}{d t}=2 W_{1}-W_{3}= & 2 k_{1}(T)\left(1-n_{\mathrm{O}}-n_{\mathrm{CO}}\right)^{2} \\
& -k_{3}(T) n_{\mathrm{O}} n_{\mathrm{CO}}, \\
\frac{d n_{\mathrm{CO}}}{d t}=W_{2}-W_{3}= & k_{2}(T)\left(1-n_{\mathrm{O}}-n_{\mathrm{CO}}\right)-k_{3}(T) n_{\mathrm{O}} n_{\mathrm{CO}} .
\end{aligned}
$$

The rate constant of the process $(2.1)$ is assumed in the Arrhenius form

$$
k_{1}(T)=k_{1}\left(T_{B}\right) \exp \left[\frac{E}{R}\left[\frac{1}{T_{B}}-\frac{1}{T}\right]\right],
$$

where $T_{B}$ is room temperature, $E$ is the activation energy for the dissociative adsorption of the $\mathrm{O}_{2}$ molecule (assumed independent of the coverage), and $R$ is the gas constant. Also, processes (2.2) and (2.3) are not considered activates and, therefore, $k_{2}(T)=k_{2}\left(T_{B}\right)$ and $k_{3}(T)=k_{3}\left(T_{B}\right)$.

If thermal diffusivity of the surface is much higher than that of the surroundings, the surface temperature, due to adsorption and reaction heats, can be different from the room temperature, thus affecting the process rates. Equations (1) and (2) must be completed with an equation for the surface temperature,

$$
C \frac{d T}{d t}=-L\left(T-T_{B}\right)+\sum_{i=1}^{3} \Delta H_{i} W_{i},
$$

where $C$ is the heat capacity of the system, $L / C$ ( $L$ is approximately equal to thermal conductivity times a geometric factor) is the relaxation rate of $T$ to $T_{B}$, and $\Delta H_{i}$ are the reaction heats of the processes (2.1)-(2.3), including a suitable geometric factor.

Kinetic equations (2.4)-(2.6) can be written in terms of the dimensionless constants and variables

$$
\begin{aligned}
& z=\frac{T}{T_{B}}-1, \quad \tau=k_{3} t, \quad \varepsilon=\frac{E}{R T_{B}}, \quad \gamma=\frac{L}{k_{3} C}, \\
& a=2 \frac{k_{1}\left(T_{B}\right)}{k_{3}\left(T_{B}\right)}, \quad b=\frac{k_{2}\left(T_{B}\right)}{k_{3}\left(T_{B}\right)}, \\
& h_{1}=\frac{\Delta H_{1}}{2 C T_{B}}, \quad h_{2}=\frac{\Delta H_{2}}{C T_{B}}, \quad q=\frac{\Delta Q}{C T_{B}},
\end{aligned}
$$

where $\Delta Q=\Delta H_{1} / 2+\Delta H_{2}+\Delta H_{3}>0$ is the net reaction heat. Moreover, for simplicity, $h_{1}=h_{2}=h$ is assumed.

Kinetic equations are

$\frac{d n_{\mathrm{O}}}{d \tau}=a g(z)\left(1-n_{\mathrm{O}}-n_{\mathrm{CO}}\right)^{2}-n_{\mathrm{O}} n_{\mathrm{CO}}$,

$\frac{d n_{\mathrm{CO}}}{d \tau}=b\left(1-n_{\mathrm{O}}-n_{\mathrm{CO}}\right)-n_{\mathrm{O}} n_{\mathrm{CO}}$,

$$
\begin{aligned}
\frac{d z}{d \tau}=-\gamma z+h[ & a g(z)\left(1-n_{\mathrm{O}}-n_{\mathrm{CO}}\right)^{2}+b\left(1-n_{\mathrm{O}}-n_{\mathrm{CO}}\right) \\
& \left.-2 n_{\mathrm{O}} n_{\mathrm{CO}}\right]+q n_{\mathrm{O}} n_{\mathrm{CO}}
\end{aligned}
$$

where $g(z)=\exp [\varepsilon z /(z+1)]$.

Equations (2.7)-(2.9) can give rise to oscillatory states for certain values of the parameters $\Gamma=\{a, b, \varepsilon, \gamma, h, q\}$.

Four steady solutions, $x_{i}=\left\{n_{\mathrm{O}}^{\mathrm{st}}, n_{\mathrm{CO}}^{\text {st }}, z^{\text {st }}\right\}$, are obtained from $d n_{\mathrm{O}} / d \tau=d n_{\mathrm{CO}} / d \tau=d z / d \tau=0$, which are denoted

$$
\begin{aligned}
& x_{+1}=(1,0,0), \\
& x_{-1}=(0,1,0), \\
& x_{0+}=\left(n_{\mathrm{O}}^{\mathrm{st}+}, n_{\mathrm{CO}}^{\mathrm{st}+}, z^{\mathrm{st}+}\right), \\
& x_{0-}=\left(n_{\mathrm{O}}^{\mathrm{st}-}, n_{\mathrm{CO}}^{\mathrm{st}-}, z^{\mathrm{st}-}\right),
\end{aligned}
$$

with $\quad n_{\mathrm{O}}^{\mathrm{st} \pm}=\frac{1}{2}\left(1-v_{0} \pm u_{0}\right), \quad n_{\mathrm{CO}}^{\mathrm{st} \pm}=\frac{1}{2}\left(1-v_{0} \mp u_{0}\right), \quad z^{\text {st } \pm}$ $=(q / \gamma) n_{\mathrm{O}}^{\mathrm{st} \pm} n_{\mathrm{CO}}^{\mathrm{st} \pm} \quad$ where $\quad v_{0}=\left[b / a g\left(z^{\text {st }}\right)\right]$ and $u_{0}=\left[\left(1-v_{0}\right)^{2}-4 b v_{0}\right]^{1 / 2}$.

Solutions $x_{0+}$ and $x_{0-}$ are for the meaningful range $\left(0<n_{\mathrm{O}}^{\text {st }}<1,0<n_{\mathrm{CO}}^{\text {st }}<1,0<n_{\mathrm{O}}^{\text {st }}+n_{\mathrm{CO}}^{\text {st }}<1\right)$ for values of $v_{0}<1$ and $4 b v_{0}<\left(1-v_{0}\right)^{2}$. Solutions $x_{0+}$ and $x_{0-}$ are symmetrical with each other.

The stability analysis is performed in the usual way, from the eigenvalues of the stability matrix. ${ }^{22} x_{+1}$ is a saddle point and $x_{-1}$ is a stable node (corresponding to the poisoning of the surface by $\mathrm{CO}$ at room temperature); $x_{0+}$ and $x_{0-}$ can be unstable, marginally stable, or stable, depending on the values of the parameter $\Gamma$.

With the aim of studying the oscillatory behavior of the system and the existence of limit cycles three representative cases have been analyzed.

(i) $q=0$ and $\gamma \rightarrow \infty$. For this case only Eqs. (2.7) and (2.8) must be considered because as $\gamma \rightarrow \infty$ the system relaxes instantaneously to room temperature $(z=0)$.

Now $x_{0-}$ is a saddle point and $x_{0+}$ is a stable node or a spiral point (state marginally stable), depending on the values of $\Gamma=\{a, b\}$. As can be seen, there are no selfsustained oscillations.

The Runge-Kutta ${ }^{23}$ method has been applied for different initial conditions. In Fig. 1, for $a=1$ and $b=0.2$, some trajectories in the phase space $n_{\mathrm{O}}-n_{\mathrm{CO}}$ are shown. Steady solutions are $x_{0+}=(0.7464,0.0536)$ (stable node), $x_{0-}=(0.0536,0.7464)$ (saddle point), $x_{+1}=(1,0)$ (saddle point), and $x_{-1}=(0,1)$ (stable node). The phase space is clearly split into two basins, corresponding to $x_{0+}$ and $x_{-1}$, respectively. The separatrix crosses the saddle point $x_{0-}$.

(ii) $q=0$ and $\gamma$ finite. In this case, the steady solutions are only functions of $\Gamma=\{a, b\}$, since $z^{\text {st }}=0$, and can be obtained as in case (i). However, for analyzing stability of solutions the set of parameters $\Gamma=\{a, b, \varepsilon, \gamma, h\}$ must be 


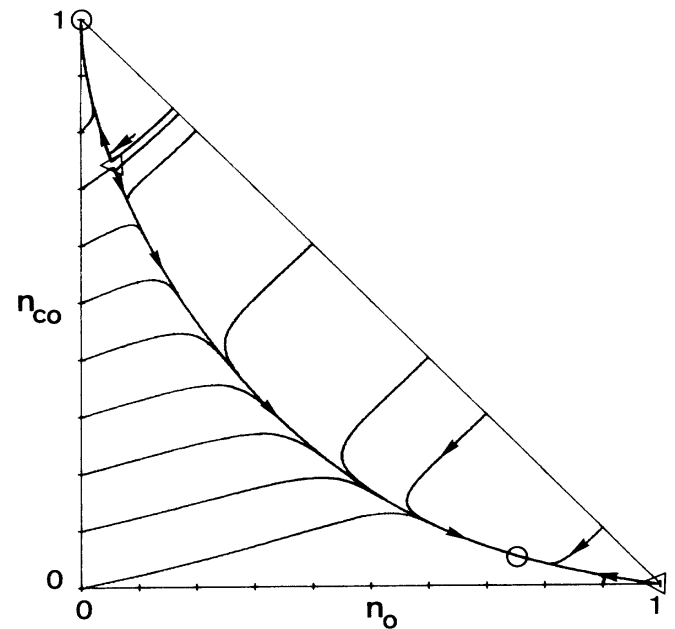

FIG. 1. Trajectories on the phase plane $n_{\mathrm{O}}-n_{\mathrm{CO}}$ for the case $q=0$ and $\gamma \rightarrow \infty$, with $a=1, b=0.2$. $\bigcirc$ denotes stable node and $\triangle$ saddle point.
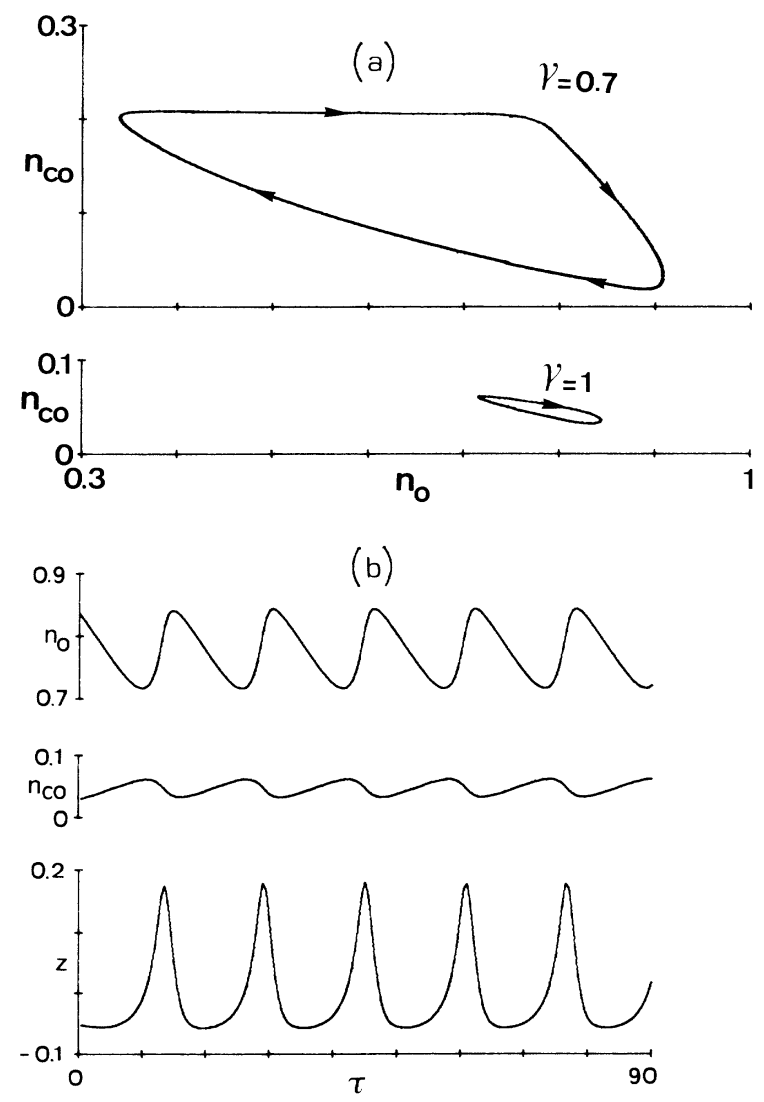

FIG. 2. (a) Projection on the $n_{\mathrm{O}}-n_{\mathrm{CO}}$ plane of several limit cycles: $\gamma=0.7$ and 1 both with $a=1, b=0.2, \varepsilon=10, h=5$, $q=0$. The unstable focus is located in the state $n_{\mathrm{O}}=0.7464$, $n_{\mathrm{CO}}=0.0536, z=0$ for both cases. (b) Time evolution of temperature and concentrations for $a=1, b=0.2, \varepsilon=10, h=5$, $q=0, \gamma=1$. considered.

Steady solution $x_{0-}$ is also a saddle point, and $x_{-1}$ a stable node, and the trajectories starting from points in the basin of $x_{-1}$ tend to $x_{-1}$; the remaining trajectories tend to the stable node $x_{0+}$ or, if $x_{0+}$ is unstable, come near to a limit cycle. For $0<\gamma<\gamma_{c}$ (with the other parameter fixed) limit cycles increase in size as $\gamma$ decreases in relation to $\gamma_{c}$. Thus, for $a=1, b=0.2, \varepsilon=10$, and $h=5, \gamma_{c}$ is 1.11 and limit cycles can be found for the range $0<\gamma<1$.11. Steady solutions for $a=1$ and $b=0.2$ (they do not depend on $\varepsilon, h$, and $\gamma$ ) are $x_{+1}=(1,0,0)$, $x_{-1}=(0,1,0), \quad x_{0+}=(0.7464,0.0536,0), \quad$ and $x_{0-}$ $=(0.0536,0.7464,0)$. In general, $x_{0+}$ is an unstable focus, marginally stable state, stable focus, and stable node as $\gamma$ increases, for the above values of the other parameters.

In Fig. 2(a) two limit cycles are shown for $\gamma=0.7$ and 1 (both for $a=1, b=0.2, \varepsilon=10, h=5, q=0$ ). In Fig. 2(b), oscillations in concentrations and temperature are shown for $\gamma=1$.

(iii) $q \neq 0$ and $\gamma$ finite. Now, although an analytical solution is not possible and numerical methods must be applied, the distribution of singular points, stability, and separatrix are similar to those of case (ii).

An analysis has been performed considering different values of $h$ and $\gamma$, choosing $q / h=2$ and fixing the other parameters. Now, $x_{0+}$ is a stable node, stable focus, marginally stable state, and an unstable focus as $\gamma$ increases. For certain values of $\gamma, x_{0+}$ and $x_{0-}$ disappear, $x_{+1}$ and $x_{-1}$ being the only steady states.

In Fig. 3 a limit cycle is shown for $a=0.0027, b=0.8$,
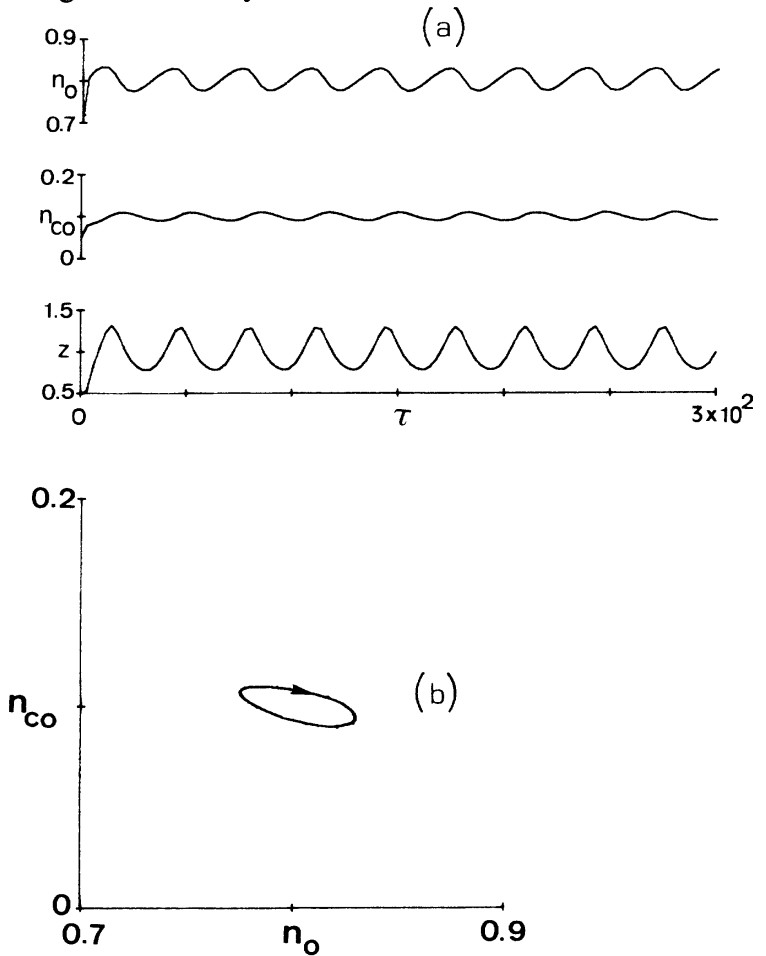

FIG. 3. Oscillatory state $n_{\mathrm{O}}=0.8003, n_{\mathrm{CO}}=0.0998$, $z=0.9988$ obtained for $a=0.0027, b=0.8, \varepsilon=16, h=12.5$, $q=25, \gamma=2$. Case of large $h$ (large surface to volume ratio). Temporary oscillations are shown in (a) and the projection on the $n_{\mathrm{O}}-n_{\mathrm{CO}}$ plane of the limit cycle obtained is shown in (b). 
$\varepsilon=16, h=12.5, q=25, \gamma=2$. The temperature and concentration oscillations are shown in Fig. 3(a) and projection on the $n_{\mathrm{O}}-n_{\text {CO }}$ plane of the limit cycle obtained is drawn in Fig. 3(b). Here $h$ is large (large surface to volume ratio).

If $\gamma$ is changed, for the same values of the other parameters, limit cycles can be obtained for the range $1.70 \leq \gamma<5.06, x_{0+}$ and $x_{0-}$ disappearing for $\gamma=5.06$. Temperature decreases as $\gamma$ increases, changing between 605 and $548 \mathrm{~K}$, with $T_{B}=298 \mathrm{~K}$.

For $h$ small (Fig. 4), calculations have been performed for $a=0.002, b=0.05, \varepsilon=20, h=0.5, q=1, \gamma=0.1$. Limit cycles appear for $0.07 \leq \gamma<0.15$, temperature changing between 377 and $365 \mathrm{~K}$, with $T_{B}=298 \mathrm{~K}$.

As can be seen, frequency and amplitude of oscillations depend on the case considered. For elevated temperature ( $T \geq 550 \mathrm{~K}, h$ large) amplitudes and periods are smaller than for lower temperature ( $T \leq 380 \mathrm{~K}, h$ small).

\section{REACTIVE DESORPTION. SCA}

Now we assume reaction takes place according to five elemental mechanisms:

$$
\mathrm{O}_{2}(g)+2 V(s) \rightarrow 2 \mathrm{O}(s),
$$
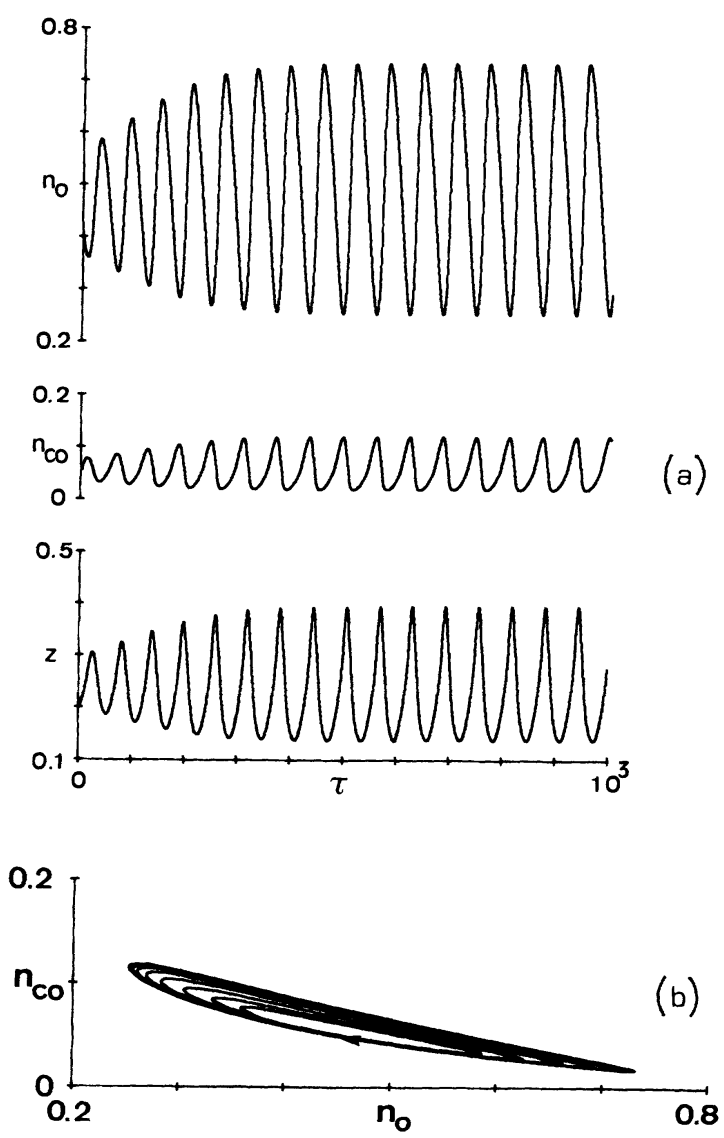

FIG. 4. Oscillatory state $n_{\mathrm{O}}=0.4568, n_{\mathrm{CO}}=0.0536$, $z=0.2448$ obtained for $a=0.002, b=0.05, \varepsilon=20, h=0.5$, $q=1, \gamma=0.1$. Case of small $h$ (small surface to volume ratio). Time display of the oscillations is shown in (a) and the projection on the $n_{\mathrm{O}}-n_{\text {CO }}$ plane of the limit cycle obtained is shown in (b).

$$
\begin{aligned}
& \mathrm{CO}(g)+V(s) \rightarrow \mathrm{CO}(s), \\
& \mathrm{CO}(s)+\mathrm{O}(s) \rightarrow \mathrm{CO}_{2}(g)+2 V(s), \\
& 2 \mathrm{O}(s) \rightarrow \mathrm{O}_{2}(g)+2 V(s), \\
& \mathrm{CO}(s) \rightarrow \mathrm{CO}(g)+V(s),
\end{aligned}
$$

thus extending the previous model analyzed in Sec. II, by including desorption processes of oxygen (3.4) and carbon monoxide (3.5) from surface.

Now kinetic equations are

$$
\begin{aligned}
& \frac{d n_{\mathrm{O}}}{d \tau}=a g(z)\left[1-n_{\mathrm{O}}-n_{\mathrm{CO}}\right]^{2}-n_{\mathrm{O}} n_{\mathrm{CO}}-a^{\prime} n_{\mathrm{O}}^{2} \\
& \frac{d n_{\mathrm{CO}}}{d \tau}=b\left(1-n_{\mathrm{O}}-n_{\mathrm{CO}}\right)-n_{\mathrm{O}} n_{\mathrm{CO}}-b^{\prime} g^{\prime}(z) n_{\mathrm{CO}}, \\
& \frac{d z}{d \tau}=-\gamma z+h\left[a g(z)\left(1-n_{\mathrm{O}}-n_{\mathrm{CO}}\right)^{2}\right. \\
& \left.+b\left(1-n_{\mathrm{O}}-n_{\mathrm{CO}}\right)-2 n_{\mathrm{O}} n_{\mathrm{CO}}\right] \\
& +q n_{\mathrm{O}} n_{\mathrm{CO}}-h^{\prime}\left[a^{\prime} n_{\mathrm{O}}^{2}+b^{\prime} g^{\prime}(z) n_{\mathrm{CO}}\right],
\end{aligned}
$$

where $a^{\prime}$ and $b^{\prime}$ are the ratios of the preexponential factors in the Arrhenius form of the processes (3.4)-(3.3) and (3.5)-(3.3), respectively; $\varepsilon^{\prime}$ is a quantity proportional to the activation energy of the process $(3.5) ; h^{\prime}$ is a quantity proportional to the ratio of the reaction heats in processes (3.4) and (3.5) to the heat capacity of the system; and $g^{\prime}(z)=\exp \left[\varepsilon^{\prime} z /(z+1)\right]$. The other parameters have been defined in Sec. II. Now the set of parameters concerning the problem is $\Gamma=\left\{a, b, a^{\prime}, b^{\prime}, \varepsilon, \varepsilon^{\prime}, \gamma, h, h^{\prime}, q\right\}$.

The previous model analyzed in Sec. II, which does not take into account desorption, gives rise to oscillatory states for certain values of the parameters. To analyze how desorption influences oscillations, case (ii) in Sec. II ( $q=0, \gamma$ finite), with $a=1, b=0.2, \varepsilon=10, h=5$, is studied. Then the problem depends on the parameters $a^{\prime}, b^{\prime}$, $\varepsilon^{\prime}$, and $h^{\prime}$, corresponding to desorption processes, and $\gamma$.

(i) $a^{\prime}=0, b^{\prime} \neq 0$. This case assumes that oxygen desorption does not take place. Experimental ${ }^{24-26}$ results show that for a temperature lower than $600 \mathrm{~K}$ the reaction kinetics is governed essentially by the $\mathrm{CO}$ coverage. Desorption rate of oxygen is negligible and $\mathrm{CO}$ coverage is governed mainly by the parameters proportional to rates of $\mathrm{CO}$ adsorption and desorption ( $b$ and $b^{\prime}$, respectively).

Steady state $x_{+1}=(1,0,0)$ always exists and is a saddle point. Additionally, up to three steady solutions can be obtained, depending on the values of $h^{\prime}$.

(a) $h^{\prime}=h$. For this case $z^{\text {st }}=0$ and the other steady solutions depend on $\Gamma=\left\{a, b, b^{\prime}\right\}$. The values of $a=1$ and $b=0.2$ being fixed, three steady solutions are obtained from $b^{\prime}=0$ (there is no $\mathrm{CO}$ desorption) up to $b^{\prime}=0.011$. For $b^{\prime}=0.012$ two of these solutions disappear.

When $\quad b^{\prime}=0.01$, steady solutions are $x_{-1}$ $=(0.0056,0.9223,0), \quad x_{0-}=(0.0236,0.8359,0), \quad$ and $x_{0+}=(0.7507,0.0519,0)$. If $\varepsilon^{\prime}$ is fixed and the values of $\gamma(\gamma>0)$ are increasing, $x_{-1}$ is a stable focus or node, $x_{0-}$ is a saddle point, and $x_{0+}$ can be an unstable focus (giving rise to a limit cycle), marginally stable state, stable 
focus, and stable node. For $b^{\prime}=0.012, x_{-1}$ and $x_{0-}$ disappear and $x_{0+}=(0.7516,0.0517,0)$.

Now, the formation of a CO packed structure, $x_{-1}$, limits the reaction steady rate by an end, and the formation of a state where oxygen atoms are closely bound (oxygen adsorption predominates), $x_{0+}$, limits it at the other end.

Thus the trajectories in the phase space tend to $x_{-1}$ or $x_{0+}$ and if $x_{0+}$ is not stable they come near a limit cycle, for $0<\gamma<\gamma_{c}$. The size of the limit cycle decreases as $\gamma$ increases. For $\varepsilon^{\prime}=0.10$ there are limit cycles for the range $0<\gamma<1.08$; for $\varepsilon^{\prime}=20$ in $0<\gamma<1.09$, and for $\varepsilon^{\prime}=50, \quad 0<\gamma<1.10$, i.e., the value of $\varepsilon^{\prime}$ scarcely influences the value of $\gamma_{c}$. In Fig. 5 a limit cycle is shown, for $b^{\prime}=0.01, \varepsilon^{\prime}=5$, and $\gamma=0.7$.

(b) $h^{\prime}<h$. For values $h^{\prime} \geq 4.5$, with $b^{\prime}=0.1, \varepsilon^{\prime}=5$, and $\gamma=0.7$, four steady solutions can be found, $x_{+1}, x_{-1}$, $x_{0-}, x_{0+}$, and their characteristics are similar to those of case (a). However, unlike case $h=h^{\prime}$ (where the system relaxed to room temperature, $z^{\text {st }}=0$ ), now the desorption mechanism makes $z^{\text {st }}>0$. Thus, for $h^{\prime}=4.5$ and the values of the other parameters fixed, $x_{+1}=(1,0,0)$ (saddle point), $x_{-1}=(0.0092,0.9025,0.0067)$ (stable focus), $x_{0-}=(0.0155,0.8718,0.0064) \quad$ (saddle point), and $x_{0+}=(0.7517,0.0516,0.0004)$ (unstable focus).

For $h^{\prime}<4.5, x_{-1}$ and $x_{0-}$ disappear. In Fig. 6 a limit
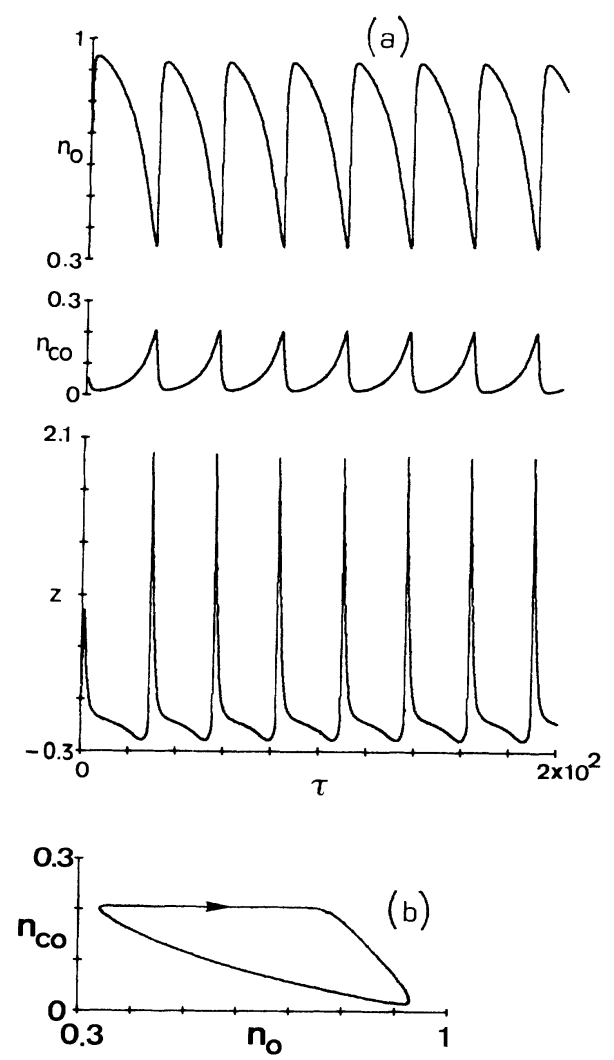

FIG. 5. Oscillatory state $n_{\mathrm{O}}=0.7507, n_{\mathrm{CO}}=0.0519, z=0$ obtained for $a=1, b=0.2, \varepsilon=10, h=5, q=0, \gamma=0.7, a^{\prime}=0$, $b^{\prime}=0.01, \varepsilon^{\prime}=5, h^{\prime}=5$. Case $h=h^{\prime}$. Temporary oscillations of coverage fractions and temperature are shown in (a). The projection on the $n_{\mathrm{O}}-n_{\text {CO }}$ plane of the limit cycle is in (b). cycle corresponding to this case is shown, with $h^{\prime}=3$, $b^{\prime}=0.1, \quad \varepsilon^{\prime}=5$, and $\gamma=0.7$, resulting in $x_{0+}$ $=(0.8057,0.0350,0.0105)$. If $\gamma$ varies with the other parameters fixed, limit cycles can be obtained for the range $0<\gamma<0.90$.

(ii) $a^{\prime} \neq 0, b^{\prime}=0$. For this case the steady state $x_{-1}=(0,1,0)$ is always obtained; it is a stable node. Depending on the values of $h^{\prime}$, two additional steady solutions can be obtained, but the state $x_{+1}=(1,0,0)$ never appears. When $h^{\prime}=h$ or $h^{\prime}<h$ there are limit cycles for the range $0<\gamma<\gamma_{c}$ (with a suitable value for $\gamma_{c}$ ) but $z^{\text {st }}=0$ for the former case and $z^{\text {st }}>0$ for the latter.

(iii) $a^{\prime} \neq 0, b^{\prime} \neq 0$. Now, some of the states $x_{+1}=(1,0,0)$ or $x_{-1}=(0,1,0)$ are not obtained, unlike the previous cases. With regard to existence and characteristics of limit cycles, results are similar to those previously obtained.

Summarizing, with regard to oscillations, when desorption exists, the behavior of the system is essentially similar to the case when desorption is not operative. The most remarkable feature is $z^{\text {st }}>0$ (for $h^{\prime}<h$ ), i.e., temperature fluctuates around a temperature greater than room temperature.

\section{DOUBLET CLOSURE APPROXIMATION}

In order to analyze the influence of the closure approximation applied on the existence of multiplicity and, espe-
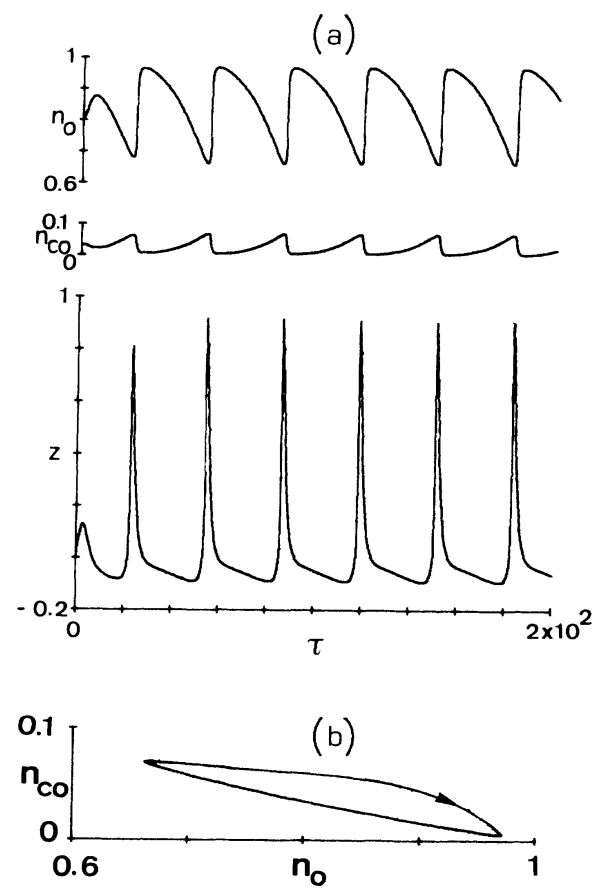

FIG. 6. Oscillatory state $n_{\mathrm{O}}=0.8057, n_{\mathrm{CO}}=0.0350$, $z=0.0105$ obtained for $a=1, b=0.2, \varepsilon=10, h=5, q=0$, $\gamma=0.7, a^{\prime}=0, b^{\prime}=0.1, \varepsilon^{\prime}=5, h^{\prime}=3$. Case $h>h^{\prime}$. Temporary oscillations are shown in (a) and the projection on the $n_{\mathrm{O}}{ }^{-n_{\mathrm{CO}}}$ plane of the limit cycle in (b). 
cially, on oscillations, in this section kinetic equations have been obtained for the kinetic model of Sec. II by applying a closure approximation consisting of closing the hierarchy of kinetic equations at the level of doublets (DCA). In general, the densities of clusters including more than two sites are written in terms of densities of singlets, $n_{i}$, and doublets, $n_{i j}$ :

$$
n_{i j k}=\frac{n_{i j} n_{j k}}{n_{j}},
$$

where $i, j$, and $k$ can be $V$ (vacancy), $C O$ (site occupied by a carbon monoxide molecule), and $\mathrm{O}$ (site occupied by an oxygen atom).

Now we assume that the surface is a square twodimensional lattice. Following the guideline of Ref. 27, in a doublet closure approximation the state of the sur- face is determined giving the density of single particles $n_{i}$ and the density of pairs, $n_{i j}^{x}$ and $n_{i j}^{y}$, where $i, j=V, \mathrm{CO}$, $\mathrm{O}$, and $x(y)$ indicates that the pair is situated on the $x$ axis ( $y$ axis). Thus, for a carbon monoxide molecule, $\mathrm{CO}$, adsorbed on the surface, the four nearest neighbors are considered, leaving the surface when a neighbor oxygen atom is found to react, forming a carbon dioxide molecule. For an oxygen molecule, $\mathrm{O}_{2}$, adsorbed on the surface, its six nearest neighbors are considered; one oxygen atom leaves the surface when meeting a neighbor carbon monoxide molecule to form a carbon dioxide molecule. By dependence relationship among the variables $n_{i}, n_{i j}^{x}$, and $n_{i j}^{y}$, the number of independent variables can be reduced. Choosing as independent variables $n_{\mathrm{O}}, n_{\mathrm{CO}}, n_{\mathrm{O}-\mathrm{O}}^{x}$, $n_{\mathrm{O}-\mathrm{CO}}^{x}, n_{\mathrm{CO}-\mathrm{CO}}^{x}$, and the scaled temperature $z$, kinetic equations are

$$
\begin{aligned}
& \frac{d n_{\mathrm{O}}}{d \tau}=a g(z)\left[1-2 n_{\mathrm{O}}-2 n_{\mathrm{CO}}+n_{\mathrm{O}-\mathrm{O}}+2 n_{\mathrm{O}-\mathrm{CO}}+n_{\mathrm{CO}-\mathrm{CO}}\right]-n_{\mathrm{O}-\mathrm{CO}}, \\
& \frac{d n_{\mathrm{CO}}}{d \tau}=b\left(1-n_{\mathrm{O}}-n_{\mathrm{CO}}\right)-n_{\mathrm{O}-\mathrm{CO}} \\
& \frac{d n_{\mathrm{O}-\mathrm{O}}}{d \tau}=\frac{1}{2} a g(z)\left[1-2 n_{\mathrm{O}}-2 n_{\mathrm{CO}}+n_{\mathrm{O}-\mathrm{O}}+2 n_{\mathrm{O}-\mathrm{CO}}+n_{\mathrm{CO}-\mathrm{CO}}\right]\left[\frac{1}{2}+\frac{3\left(n_{\mathrm{O}}-n_{\mathrm{O}-\mathrm{O}}-n_{\mathrm{O}-\mathrm{CO}}\right)}{\left(1-n_{\mathrm{O}}-n_{\mathrm{CO}}\right)}\right]-\frac{3 n_{\mathrm{O}-\mathrm{O}} n_{\mathrm{O}-\mathrm{CO}}}{2 n_{\mathrm{O}}}, \\
& \frac{d n_{\mathrm{O}-\mathrm{CO}}}{d \tau}=\frac{3 a g(z)}{4\left(1-n_{\mathrm{O}}-n_{\mathrm{CO}}\right)}\left[1-2 n_{\mathrm{O}}-2 n_{\mathrm{CO}}+n_{\mathrm{O}-\mathrm{O}}+2 n_{\mathrm{O}-\mathrm{CO}}+n_{\mathrm{CO}-\mathrm{CO}}\right]\left[n_{\mathrm{CO}}-n_{\mathrm{O}-\mathrm{CO}}-n_{\mathrm{CO}-\mathrm{CO}}\right] \\
& +b\left[n_{\mathrm{O}}-n_{\mathrm{O}-\mathrm{O}}-n_{\mathrm{O}-\mathrm{CO}}\right]-\frac{n_{\mathrm{O}-\mathrm{CO}}}{4}\left[1+\frac{3 n_{\mathrm{O}-\mathrm{CO}}}{n_{\mathrm{O}}}+\frac{3 n_{\mathrm{O}-\mathrm{CO}}}{n_{\mathrm{CO}}}\right] \text {, } \\
& \frac{d n_{\mathrm{CO}-\mathrm{CO}}}{d \tau}=2 b\left[n_{\mathrm{CO}}-n_{\mathrm{O}-\mathrm{CO}}-n_{\mathrm{CO}-\mathrm{CO}}\right]-\frac{3 n_{\mathrm{O}-\mathrm{CO}} n_{\mathrm{CO}-\mathrm{CO}}}{2 n_{\mathrm{CO}}} \text {, } \\
& \frac{d z}{d \tau}=-\gamma z+h\left\{a g(z)\left[1-2 n_{\mathrm{O}}-2 n_{\mathrm{CO}}+n_{\mathrm{O}-\mathrm{O}}+2 n_{\mathrm{O}-\mathrm{CO}}+n_{\mathrm{CO}-\mathrm{CO}}\right]+b\left(1-n_{\mathrm{O}}-n_{\mathrm{CO}}\right)-2 n_{\mathrm{O}-\mathrm{CO}}\right\}+q n_{\mathrm{O}-\mathrm{CO}} \text {, }
\end{aligned}
$$

where $n_{\mathrm{O}-\mathrm{O}}, n_{\mathrm{O}-\mathrm{CO}}$, and $n_{\mathrm{CO}-\mathrm{CO}}$ are the densities of pairs $\mathrm{O}-\mathrm{O}, \mathrm{O}-\mathrm{CO}$, and CO-CO, respectively; $\Gamma=\{a, b, \varepsilon, \gamma, h, q\}$ is the set of relevant parameters of the model.

In general, four steady solutions, $x=\left(n_{\mathrm{O}}\right.$, $\left.n_{\mathrm{CO}}, n_{\mathrm{O}-\mathrm{O}}, n_{\mathrm{O}-\mathrm{CO}}, n_{\mathrm{CO}-\mathrm{CO}}, z\right)$, are obtained, at most; they are labeled, for analogy with Secs. II and III, as $x+1$ $=(1,0,1,0,0,0), \quad x_{-1}=(0,1,0,0,1,0), \quad x_{0+}=\left(n_{\mathrm{O}}^{\text {st }+}\right.$, $\left.n_{\mathrm{CO}}^{\mathrm{st}+}, n_{\mathrm{O}-\mathrm{O}}^{\mathrm{st}+}, n_{\mathrm{O}-\mathrm{CO}}^{\mathrm{st}+}, n_{\mathrm{CO}-\mathrm{CO}}^{\mathrm{st}+}, z^{\mathrm{st}+}\right)$, with $n_{\mathrm{O}}^{\mathrm{st}+}>n_{\mathrm{CO}}^{\mathrm{st}+}$, and $x_{0-}=\left(n_{\mathrm{O}}^{\mathrm{st}-}, n_{\mathrm{CO}}^{\mathrm{st}-}, n_{\mathrm{O}-\mathrm{O}}^{\mathrm{st}}, n_{\mathrm{O}-\mathrm{CO}}^{\mathrm{st}-}, n_{\mathrm{CO}-\mathrm{CO}}^{\mathrm{st}-} z^{\mathrm{st}-}\right)$, with $n_{\mathrm{O}}^{\mathrm{st}-}$ $<n_{\mathrm{CO}}^{\text {st }}$; now $x_{0-}$ is not symmetrical with $x_{0+}$. Thus the number of steady solutions in the DCA is the same as in the SCA.

The previous cases analyzed in Sec. II are now considered: $q=0, \gamma \rightarrow \infty ; q=0, \gamma$ finite; $q \neq 0, \gamma$ finite.

The results obtained are qualitatively similar to those of Sec. II, but there are some quantitative differences. Thus the range $0<\gamma<\gamma_{c}$ allowing oscillations is shortened; for example, in the case $q=0, \gamma$ finite, for $a=1$, $b=0.2, \varepsilon=10$, and $h=5, \gamma_{c}=0.34$ for the DCA, but $\gamma_{c}=1.11$ for the SCA. Some quantitative differences for the values of $n_{\mathrm{O}}, n_{\mathrm{CO}}$, and so on, and reaction rate can be appreciated.

Figures 7 and 8, both with $a=0.002, b=0.05, \varepsilon=20$, $h=0.5, q=1$, and $\gamma=0.08$, show case $q \neq 0$ and $\gamma$ finite for DCA. Figure 7 shows the projection of limit cycle on the phase plane $n_{\mathrm{O}}-n_{\mathrm{CO}}$; and temperature and concentration oscillations for the same limit cycle are shown in Fig. 8. Both figures can be compared with Fig. 4, which represents an analogous case in SCA.

Summarizing, in this two-dimensional model, the kind of approximation applied does not essentially affect the results, although some quantitative differences appear. Considering the kinetic phase transitions taking place in

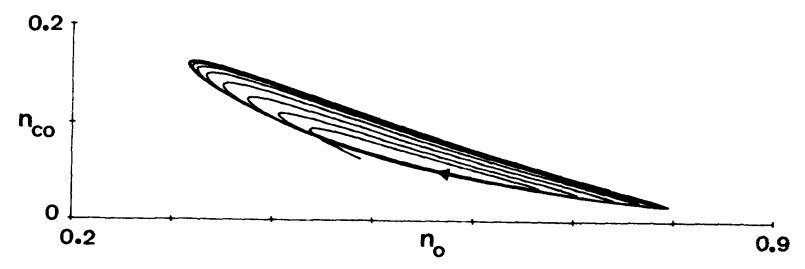

FIG. 7. Projection on the phase plane $n_{\mathrm{O}}-\boldsymbol{n}_{\mathrm{CO}}$ of the limit cycle obtained for $a=0.002, b=0.05, \varepsilon=20, h=0.5, q=1$, $\gamma=0.08$. The unstable focus is $n_{\mathrm{O}}=0.5381, n_{\mathrm{CO}}=0.0617$, $n_{\mathrm{O}-\mathrm{O}}=0.3354, n_{\mathrm{O}-\mathrm{CO}}=0.02, n_{\mathrm{CO}-\mathrm{CO}}=0.0071$, and $z=0.2501$. 
TABLE I. Kinetic phase transitions in this model.

\begin{tabular}{|c|c|c|c|c|c|}
\hline$q$ & $\gamma$ & & & \multicolumn{2}{|c|}{ Transitions } \\
\hline \multirow[t]{4}{*}{0} & \multirow[t]{2}{*}{$\infty$} & Multiplicity & YES & \multirow[t]{2}{*}{1 stable node $\leftrightarrow$} & \multirow[t]{2}{*}{2 stable nodes } \\
\hline & & Oscillations & NO & & \\
\hline & Finite & Multiplicity & YES & \multirow[b]{2}{*}{1 stable node } & \\
\hline & & $\begin{array}{l}\text { Oscillations } \\
\text { (Temp } \\
\text { around th }\end{array}$ & $\begin{array}{l}\text { YES } \\
\text { tuates } \\
\text { nperature) }\end{array}$ & & 1 stable node \\
\hline$\neq 0$ & Finite & $\begin{array}{l}\text { Multiplicity } \\
\text { Oscillations } \\
\text { (Temperatur } \\
\text { around a ter } \\
\text { than the roo }\end{array}$ & $\begin{array}{l}\text { YES } \\
\text { YES } \\
\text { reater } \\
\text { ture) }\end{array}$ & 2 stable nodes & 1 limit cycle \\
\hline
\end{tabular}

this system, depending on the "thermal" parameters $q$ and $\gamma$, Table I can be written.

\section{LINEAR CHAIN. SCA AND DCA}

Catalyzed oxidation of carbon monoxide takes place on a surface and, therefore, a two-dimensional model must be applied. However, as has been demonstrated for other cases, ${ }^{16,17,27}$ some closure approximations can introduce spurious kinetic transitions when they have been applied to one-dimensional systems. For checking this possibility in this model, the adsorption and reaction processes described in Sec. II are assumed to take place on a linear chain.

Kinetic equations applying the SCA are

$$
\begin{aligned}
& \frac{d n_{\mathrm{O}}}{d \tau}=\frac{1}{2} a g(z)\left(1-n_{\mathrm{O}}-n_{\mathrm{CO}}\right)^{2}-\frac{1}{2} n_{\mathrm{O}} n_{\mathrm{CO}} \\
& \frac{d n_{\mathrm{CO}}}{d \tau}=b\left(1-n_{\mathrm{O}}-n_{\mathrm{CO}}\right)-\frac{1}{2} n_{\mathrm{O}} n_{\mathrm{CO}}, \\
& \frac{d z}{d \tau}=-\gamma z+h\left(\frac{1}{2} a g(z)\left(1-n_{\mathrm{O}}-n_{\mathrm{CO}}\right)^{2}\right. \\
& \left.\quad+b\left(1-n_{\mathrm{O}}-n_{\mathrm{CO}}\right)-n_{\mathrm{O}} n_{\mathrm{CO}}\right)+\frac{q}{2} n_{\mathrm{O}} n_{\mathrm{CO}}
\end{aligned}
$$

and the ones resulting from the DCA are

$$
\begin{aligned}
& \frac{d n_{\mathrm{O}}}{d \tau}=\frac{1}{2} a g(z)\left[1-2 n_{\mathrm{O}}-2 n_{\mathrm{CO}}+n_{\mathrm{O}-\mathrm{O}}+2 n_{\mathrm{O}-\mathrm{CO}}+n_{\mathrm{CO}-\mathrm{CO}}\right]-\frac{1}{2} n_{\mathrm{O}-\mathrm{CO}} \\
& \frac{d n_{\mathrm{CO}}}{d \tau}=b\left(1-n_{\mathrm{O}}-n_{\mathrm{CO}}\right)-\frac{1}{2} n_{\mathrm{O}-\mathrm{CO}} \\
& \frac{d n_{\mathrm{O}-\mathrm{O}}}{d \tau}=\frac{1}{2} a g(z)\left[1-2 n_{\mathrm{O}}-2 n_{\mathrm{CO}}+n_{\mathrm{O}-\mathrm{O}}+2 n_{\mathrm{O}-\mathrm{CO}}+n_{\mathrm{CO}-\mathrm{CO}}\right]\left[\frac{1}{2}+\frac{\left(n_{\mathrm{O}}-n_{\mathrm{O}-\mathrm{O}}-n_{\mathrm{O}-\mathrm{CO}}\right)}{\left(1-n_{\mathrm{O}}-n_{\mathrm{CO}}\right)}\right]-\frac{n_{\mathrm{O}-\mathrm{O}} n_{\mathrm{O}-\mathrm{CO}}}{2 n_{\mathrm{O}}}, \\
& \frac{d n_{\mathrm{O}-\mathrm{CO}}}{d \tau}=\frac{a g(z)}{4\left(1-n_{\mathrm{O}}-n_{\mathrm{CO}}\right)}\left[1-2 n_{\mathrm{O}}-2 n_{\mathrm{CO}}+n_{\mathrm{O}-\mathrm{O}}+2 n_{\mathrm{O}-\mathrm{CO}}+n_{\mathrm{CO}-\mathrm{CO}}\right]\left[n_{\mathrm{CO}}-n_{\mathrm{O}-\mathrm{CO}}-n_{\mathrm{CO}-\mathrm{CO}}\right] \\
& +b\left(n_{\mathrm{O}}-n_{\mathrm{O}-\mathrm{O}}-n_{\mathrm{O}-\mathrm{CO}}\right)-\frac{1}{4} n_{\mathrm{O}-\mathrm{CO}}\left[1+\frac{n_{\mathrm{O}-\mathrm{CO}}}{n_{\mathrm{O}}}+\frac{n_{\mathrm{O}-\mathrm{CO}}}{n_{\mathrm{CO}}}\right], \\
& \frac{d n_{\mathrm{CO}-\mathrm{CO}}}{d \tau}=2 b\left(n_{\mathrm{CO}}-n_{\mathrm{O}-\mathrm{CO}}-n_{\mathrm{CO}-\mathrm{CO}}\right)-\frac{1}{2} \frac{n_{\mathrm{O}-\mathrm{CO}} n_{\mathrm{CO}-\mathrm{CO}}}{n_{\mathrm{CO}}}, \\
& \frac{d z}{d \tau}=-\gamma z+h\left[\frac{1}{2} a g(z)\left[1-2 n_{\mathrm{O}}-2 n_{\mathrm{CO}}+n_{\mathrm{O}-\mathrm{O}}+2 n_{\mathrm{O}-\mathrm{CO}}+n_{\mathrm{CO}-\mathrm{CO}}\right]+b\left(1-n_{\mathrm{O}}-n_{\mathrm{CO}}\right)-n_{\mathrm{O}-\mathrm{CO}}\right]+\frac{1}{2} q n_{\mathrm{O}-\mathrm{CO}} \cdot
\end{aligned}
$$



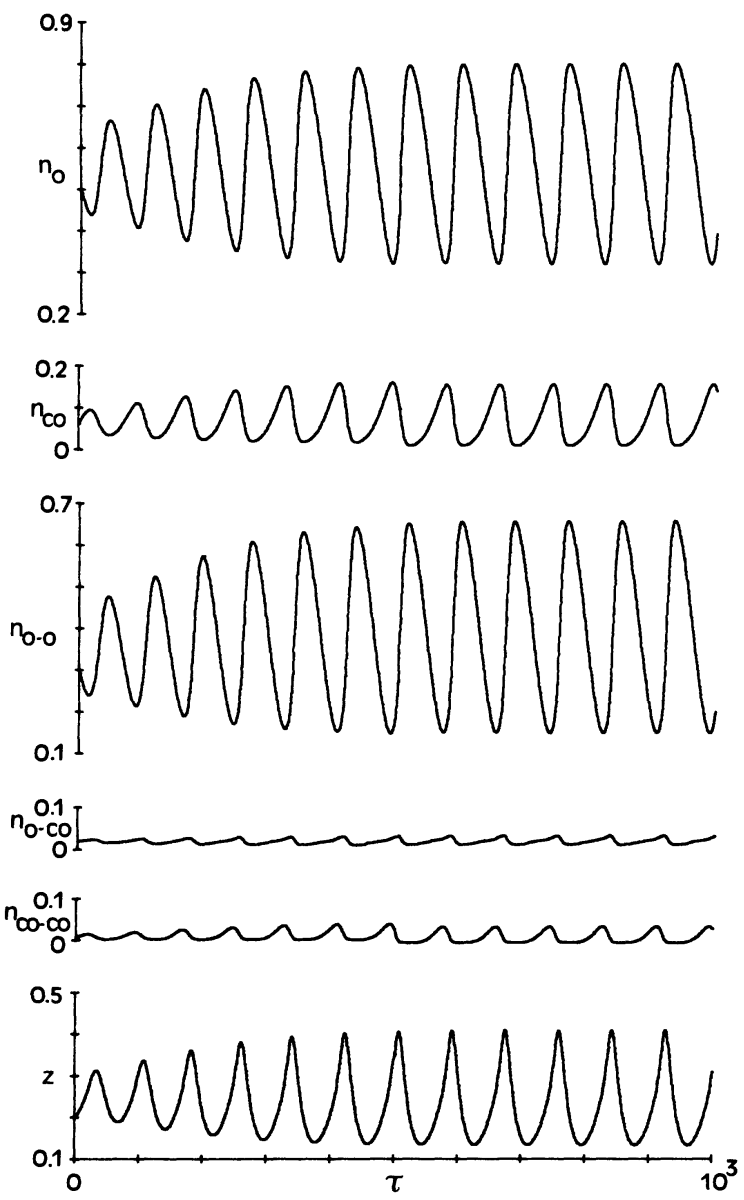

FIG. 8. Temporary oscillations of temperature and coverage fractions for $a=0.002, b=0.05, \varepsilon=20, h=0.5, q=1, \gamma=0.08$. The oscillatory state is $n_{\mathrm{O}}=0.5381, \quad n_{\mathrm{CO}}=0.0617$, $n_{\mathrm{O}-\mathrm{O}}=0.3354, n_{\mathrm{O}-\mathrm{CO}}=0.02, n_{\mathrm{CO}-\mathrm{CO}}=0.0071, z=0.2501$.

First, kinetic equations from the SCA will be analyzed.

As in the two-dimensional case, four steady solutions are obtained, $x_{+1}, x_{-1}, x_{0+}, x_{0-} ; x_{+1}$ is a saddle point and $x_{-1}$ is a stable node; $x_{0+}$ and $x_{0-}$ can be stable or

unstable, depending on the values of the parameters $\Gamma=\{a, b, \varepsilon, \gamma, h, q\}$.

Similar results to those of the two-dimensional system are obtained for the one-dimensional systems applying the SCA. However, the analysis of the kinetic equations derived from the DCA for a one-dimensional system is essentially different from that previously obtained in Sec. II and in this section for the SCA.

Effectively, for $q=0$ and $\gamma \rightarrow \infty$, only the steady states $x_{+1}=(1,0,1,0,0)$ and $x_{-1}=(0,1,0,0,1)$ are found; they can be saddle points or stable node, depending on $\Gamma=\{a, b\}$. There is no multiplicity of stable steady states.

When the possible existence of limit cycles is analyzed (for $\gamma$ finite, with $q=0$ and $q \neq 0$ ) discrepancies with the SCA also appear. A wide sweep in the framework of the parameters $\Gamma=\{a, b, \varepsilon, \gamma, h, q\}$ has been performed and, for all the analyzed cases only one stable steady state (stable node) has been obtained, corresponding to $x_{+1}=(1,0,1,0,0,0)$ or $x_{-1}=(0,1,0,0,1,0)$, depending on the values of parameter $\Gamma$.

Summarizing, the nonlinearity introduced in the model of the surface reaction formulated to study the catalyzed oxidation of carbon monoxide does not give rise to multiplicity of stable steady states or oscillations in onedimensional systems when the DCA is applied, but does when the system is two dimensional.

As in other cases of equilibrium and kinetic phase transitions, it is made clear that these take place in twodimensional systems but not in one-dimensional ones and, therefore, the transition appearing when the SCA is applied to a one-dimensional system is spurious and a consequence of the approximation applied.

\section{ACKNOWLEDGMENT}

This work was partially supported by Grant No. PB91-0608 of the DGICYT of the Spanish Government.

${ }^{1}$ L. F. Razon and R. A. Schmitz, Catal. Rev. Sci. Eng. 28, 89 (1986).

${ }^{2}$ R. M. Ziff, E. Gulari, and Y. Barshad, Phys. Rev. Lett. 56, 2553 (1986).

${ }^{3}$ R. Dickman, Phys. Rev. A 34, 4246 (1986).

${ }^{4}$ P. Fischer and U. M. Titulaer, Surf. Sci. 221, 409 (1989).

${ }^{5}$ D. Ben-Avraham, S. Redner, D.B. Considine, and P. Meakin, J. Phys. A 23, L613 (1990).

${ }^{6} \mathrm{M}$. Dumont, P. Dufour, B. Sente, and R. Dagonnier, J. Catal. 122, 95 (1990).

${ }^{7}$ J. J. Luque, Phys. Rev. A 42, 3319 (1990).

${ }^{8}$ F. Bagnoli, B. Sente, M. Dumont, and R. Dagonnier, J. Chem. Phys. 94, 777 (1991).

${ }^{9}$ M. Dumont, B. Sente, P. Dufour, and R. Dagonnier, J. Chem. Phys. 96, 4014 (1992).
10J. W. Evans, Langmuir 7, 2514 (1991).

${ }^{11}$ J. W. Evans and M. S. Miesch, Phys. Rev. Lett. 66, 833 (1991).

12J. J. Luque, Phys. Rev. A 45, 7085 (1992).

${ }^{13}$ J. J. Luque, F. Jiménez-Morales, and M. C. Lemos, J. Chem. Phys. 96, 8535 (1992).

${ }^{14}$ T. Tomé and R. Dickman, Phys. Rev. E 47, 948 (1993).

${ }^{15}$ I. Jensen and R. Dickman, Phys. Rev. E 48, 1710 (1993).

${ }^{16}$ A. Córdoba, M. C. Lemos, and J. J. Luque, J. Chem. Phys. 92, 5636 (1990).

${ }^{17}$ M. C. Lemos and A. Córdoba, J. Chem. Phys. 95, 6171 (1991).

${ }^{18}$ D. G. Vlachos, L. D. Schmidt, and R. Aris, J. Chem. Phys. 93, 8306 (1990).

${ }^{19}$ R. E. Lagos, B. C. Sales, and H. Suhl, Surf. Sci. 82, 525 (1979).

${ }^{20}$ J. E. Turner, B. C. Sales, and M. B. Maple, Surf. Sci. 103, 54 (1981). 
${ }^{21}$ B. C. Sales, J. E. Turner, and M. B. Maple, Surf. Sci. 112, 272 (1982); 114, 381 (1982).

${ }^{22}$ G. Nicolis and I. Prigogine, Self-Organization in Nonequilibrium Systems (Wiley, New York, 1977).

${ }^{23}$ S. Gill, Proc. Cambridge Philos. Soc. 47, 96 (1951).

${ }^{24}$ P. Möller, K. Wetzl, M. Eiswirth, and G. Ertl, J. Chem. Phys.
85, 5328 (1986)

${ }^{25}$ Jian-Wei He and P. R. Norton, J. Chem. Phys. 89, 1170 (1989).

${ }^{26}$ Jian-Wei He, U. Memmert, K. Griffiths, W. N. Lennard, and P. R. Norton, Surf. Sci. 202, L555 (1988).

${ }^{27}$ A. Córdoba and M. C. Lemos, J. Chem. Phys. 99, 4821 (1993). 